\title{
Approaches to studying the pathogenesis of asthma
}

\author{
Samapon Duangkham MD, Nicole Welch MD, Myrian Noella Vinan Vega MD, \\ Sima Shahbandar MD, James A. Tarbox MD
}

\begin{abstract}
A phenotype describes a group of patients who present with similar clinically observable characteristics. An endotype is a subgroup of patients who share the same pathophysiologic processes that lead to disease presentation. Asthma is a complex chronic disorder that consists of many identifiable phenotypes and two generally accepted endotypes. Understanding the characteristics of the underlying inflammation requires lung biopsies or bronchoalveolar lavage studies, which are invasive and potentially dangerous. There are ongoing investigations that study biomarkers to define asthma phenotypes and endotypes. This article reviews the potential utility of pharmacogenomics, exhaled breath condensates, and serum biomarkers in defining asthma phenotypes and endotypes.
\end{abstract}

Keywords: asthma, pathogenesis, pharmacogenetics, exhaled condensates, biomarkers

\section{INTRODUCTION}

Despite the high prevalence of asthma in the United States and the knowledge that different asthma phenotypes exist, endotypes to explain the varying phenotypes have only recently begun to be a focus of study. ${ }^{1}$ A phenotype is defined as a group of patients that present similar clinically observable characteristics, without establishing a direct etiologic relationship with a distinct pathophysiologic mechanism. An endotype, on the other hand, describes a subgroup that shares the same pathophysiologic processes that lead to the development, the progression, and the presentation of a disease. ${ }^{2}$ Biological markers (biomarkers) represent measurable indicators linking an endotype with a phenotype. ${ }^{3}$ Currently, there are two generally accepted endotypes of asthma: T-2 high (eosinophilic airway inflammation) and T-2 low (consisting of neutrophilic or paucigranulocytic inflammation). ${ }^{1,4}$

Corresponding author: James A Tarbox

Contact Information: James.Tarbox@ttuhsc.edu

DOI: 10.12746/swrccc.v10i42.987
T2-high asthma is remarkable for the presence of a high number of type 2 cytokines. These cells produce an inflammatory response which leads to severe bronchospasm. T2-low asthma is remarkable for a low number of type 2 cytokines being present. It is predominantly mediated by neutrophils and typically has more resistance to steroid treatment. ${ }^{1,4}$

Current studies on the pathogenesis of asthma present multiple problems, including but not limited to their invasive nature. Understanding the characteristics of the underlying inflammation requires lung biopsies and/or bronchoalveolar lavage studies. These tests are invasive and potentially dangerous, especially in patients with severe asthma. Investigations into different classes of biomarkers are ongoing and include omics (such as genomics, transcriptomics, proteomics, or metabolomics), microRNA (miRNA), blood biomarkers, respiratory biomarkers, and urinary biomarkers both for T2-high and T2-low asthma.

Studies using exhaled breath condensates provide an opportunity to characterize chemical compounds originating either in airways or alveolar spaces. These collections can be easily repeated in all patients and do not require invasive procedures. Currently, the value of 
exhaled nitric oxide involves its use in clinical settings to evaluate airway eosinophilia and the likely response to inhaled corticosteroids (ICS). These measurements are helpful in T-2 high asthma due to its pathogenesis involving eosinophils but unfortunately not in T2-low asthma as it is neutrophil predominant or paucigranulocytic.

Research on the natural history of asthma requires well-characterized subgroups and prolonged follow-up. Investigations on responses to drug therapy have two potential advantages: they provide immediate information about the benefit of drugs at least in certain subgroups and the mechanism of action may provide insight into the underlying pathogenesis of asthma. Drug studies which include a genetic analysis of the participants have the potential to provide information about the underlying genotype leading to the development of asthma or the genotype associated with a response to a particular drug. This review briefly discusses these topics to evaluate their potential utility in better defining asthma phenotypes and endotypes.

\section{Pharmacogenomics}

Pharmacogenetics is the study of variation in drug response among individuals due to heritable differences. ${ }^{5}$ Pharmacogenomics is "the study of how genes affect a person's response to drugs." ${ }^{\circ}$ This may be a distinction without a difference, "and both terms can be used interchangeably." ${ }^{5}$ Purists may limit the use of pharmacogenetics to "genes determining drug metabolism" while pharmacogenomics is a broader "term that encompasses all genes in the genome that may determine drug response." 6

Asthma is a disease with subgroups defined by different responses to 4 classes of asthma therapeutic drugs: beta-adrenergic receptor agonists, inhaled corticosteroids, leukotriene modifiers, and biological modifiers. ${ }^{7}$ Since there is evidence that the different responses to the first three classes of drugs have a heritable basis, responders and non-responders to each class are considered separate phenotypes. At present, we can only determine drug responsiveness after the fact following a therapeutic trial, though we have some tests that provide some indication of which patients will fall into which phenotypes. The Holy Grail of pharmacogenomics is a test or tests that predict a responsiveness phenotype before a therapeutic trial, thereby saving time, cost, and possibly unnecessary side effects.

One of the first hurdles in the pharmacogenomics of asthma is defining the response of asthma to drug therapy. Clinical responses to drug therapy, such as number of exacerbations in a time frame or subjective responses to symptom questionnaires, have been used, but these parameters may have poor correlation to quantitative measures of lung function. ${ }^{18}$ Change in forced expiratory volume in 1 second (FEV1), provocation concentration of methacholine causing a $20 \%$ drop in FEV1 $\left(\mathrm{PC}_{20}\right)$, and bronchodilator response in FEV1 following administration of a short acting beta-2-agonist are quantitative measures that have good reproducibility and good discrimination between responders and non-responders. ${ }^{7}$

Both individuals and populations demonstrate variance in the responsiveness to asthma therapy. ${ }^{8}$ The distribution in variance of repeated measures of FEV1 in single subjects approximates a normal distribution. ${ }^{8}$ Normal distributions are the result of independent interactions among many variables leading to a "random walk" distribution. Both genetic and environmental factors can lead to normal distributions. The variance in repeated measures of FEV1 in a single subject cannot be explained on a genetic basis. This source of variance is clearly environmental. Finding a good correlation between a given genotype and responsiveness in a population, however, suggests that genetic factors are among the determinants of the variance. ${ }^{8}$

There are four basic classes of asthma therapeutics: inhaled corticosteroids, beta-2 receptor agonists, leukotriene modifiers, and biological modifiers. Single nucleotide polymorphisms (SNPs) have been identified for the first three classes. ${ }^{7}$ The observed normal distribution of asthma responses to these drugs represents both a positive factor and a negative factor in determining the potential benefits of pharmacogenomics. The correlation of genotypes with responsiveness raises the possibility that genetic testing can determine responsiveness a priori, but the normal distribution of responsiveness is a testament to the large number of 
factors, including environmental factors, that will create both false positives and false negatives for these future tests.

In a study conducted in 2019 by Hernandez-Pacheco et $\mathrm{al}$ on the genome-wide association of asthma and ICS, one SNP (in a gene coding for apolipoprotein B mRNA-editing catalytic polypeptide 3 ) had a protective effect for asthma exacerbations in certain ethnic groups. ${ }^{9}$ This same SNP also correlated with improved FEV1 values with ICS treatments. ${ }^{9,10}$ Numerous other SNPs have also been identified relating to the pathogenesis of asthma; however, more investigation is needed as repeat studies have been unable to replicate the correlation between certain SNPs and treatment response..$^{10}$ If specific SNPs could be identified and correlated to clinical improvement with specific treatments, it is conceivable that evaluating for the specific SNPs could help to direct treatment in the future.

Recently, micro-RNAs (miRNA) have been found that are specific for T2-high and T2-low asthma. ${ }^{11}$ The ability to perform polymerase chain reactions (PCR) on a patient's blood sample and then to classify them into a T2-high or -low phenotype would be a simple laboratory test. The ability to differentiate endotypes at the time of diagnosis may save time and frustration by focusing on individual treatments that have shown success in that subgroup of asthma. For example, miRNAs have also been studied in relation to T2-low asthma, and it was noted that upregulation of certain miRNAs resulted in increased epithelial IL-8, steroid resistance, and pulmonary function damage. ${ }^{1}$

\section{EXHALED BREATH CONDENSATES}

Montuschi and Barnes have summarized research on the analysis of exhaled breath condensates in patients with airway inflammation. ${ }^{12}$ These studies require the subject to breathe through a condenser for approximately 15 minutes. The chemicals in the gaseous phase of exhaled breath are condensed by a cooling system; typically, 1 to $2.5 \mathrm{mls}$ of condensate are collected. Chemicals associated with oxidative stress or with inflammation are measured, and examples include 8-isoprostane, leukotrienes, prostaglandins, hydrogen peroxide, and 3-nitrotyrosine. Isoprostanes are specific for lipid peroxidation and cause contraction of bronchial smooth muscle. Exhaled 8-isoprostane is increased in patients with asthma, patients with stable chronic obstructive pulmonary diseases, and patients with cystic fibrosis. Corticosteroid treatment does not reduce the level of this chemical in patients with asthma, which might suggest that trials with other drugs are needed to determine the pathophysiologic effects of these chemicals in patients.

Kazani et al. measured lipoxin A4 (anti-inflammatory mediator) and leukotriene B4 (pro-inflammatory mediator) in healthy controls and in patients with asthma of varying severity. ${ }^{13}$ Both lipoxin A4 and leukotriene B4 were increased in asthmatics and could serve as diagnostic biomarkers. The ratio of lipoxin A4 to leukotriene B4 was decreased in patients with severe asthma. This would suggest that the anti-inflammatory response in patients with severe asthma was reduced compared to the pro-inflammatory response. Other investigators have studied the biochemical activity in asthmatic airways by analyzing the spectra from nuclear magnetic resonance spectroscopy of exhaled breath condensates or the differences in temperature. Increased temperature can be a sign of the inflammation that occurs in asthma, but research is still trying to determine a value for the normal range. ${ }^{1}$ Patients with asthma have different spectra of exhaled breath condensates than healthy controls, and this approach offers the possibility of identifying underlying endotypes in patients with asthma. ${ }^{14,15}$

Studies involving the measurement of leukotrienes, prostanoids, and nitric oxide derived chemicals in exhaled air have the potential to clarify the pathogenesis of chronic asthma, acute exacerbations in asthma, and drug effects in asthma. These studies have the potential to clarify the underlying endotype in various asthma syndromes and would complement genetic studies of drug responses in asthmatics.

\section{SERUM BIOMARKERS}

Serum biomarkers are less invasive, are easy to obtain, and can help differentiate patients into the asthma phenotypes and endotypes. However, they do not discriminate well between endotypes or precisely 
predict response to a T2 biologic. $^{16}$ The practical useful biomarkers should have good sensitivity and specificity with clear cut-off values and deliver reproducible results. ${ }^{17}$

Blood eosinophil counts emerged as a key biomarker to identify patients with eosinophilic asthma and are present in both patients with allergic asthma and those with late-onset eosinophilic asthma. It may be used mainly to identify patients who may respond to anti-IL-5. ${ }^{18}$ Eosinophil levels at $150 / \mu \mathrm{L}$ and $300 / \mu \mathrm{L}$ are often used as standard cut off levels for anti-IL-5, IL-5R, and IL-4Ra biological therapy, but there is no consensus about the optimal cut-off value, and it does not offer insight into function or number of eosinophils present in the lung during anti-IL-5 therapy. ${ }^{17}$ Serum $\operatorname{lgE}$, one of the first biomarkers, is a good biomarker for atopy status and useful for dosing of omalizumab, a monoclonal antibody against IgE. Total and specific $\operatorname{lgE}$ levels are often increased in patient with allergic asthma. However, the correlation with air flow obstruction and disease severity is poor. ${ }^{17}$

Serum periostin is an extracellular matrix protein produced by bronchial epithelial cells in response to stimulation with type 2 cytokines. However, clinical application is limited due to inconsistent results regarding the correlation with eosinophilic airway inflammation and ability to predict treatment response to IL-13 targeting treatment.

The T2-low endotype is less well characterized, and there are limited biomarkers for this endotype of asthma. ${ }^{18}$ Proposed biomarkers include blood neutrophil count, IL-17, IL-6, and YKL-40, a chitinase-like protein that is associated with serum neutrophilia. But they all lack universal cut-off values and are not specific to asthma. ${ }^{17}$

There are developing biomarkers, such as serum dipeptidyl peptidase (DPP-4), which was found to be better at predicting treatment response to IL-13 targeting therapy, serum eosinophilic cationic protein, which is a potential marker for diagnosing eosinophilic airway inflammation and an increased risk for exacerbations, and vascular endothelial growth factor, which was found to positively correlate with asthma severity. However, clinical application is limited as these potential novel biomarkers currently lack universal cut-off values and standard measurement assays. ${ }^{17}$

\section{Conclusion}

Asthma is recognized as a heterogeneous disease with several phenotypes and endotypes. Currently, there are biomarkers that correlate with disease severity and the risk of exacerbation, or can predict responses to treatment. However, many biomarkers are not yet available for routine clinical use because they lack validation and standardization. Further investigation is needed to identify underlying pathogenesis using biomarkers to define phenotypes and endotypes in asthma patients, which will enhance personalized management of asthma, will increase the effectiveness of management, save cost and time, and reduce side effects.

Article citation: Duangkham S, Welch N, Vinan Vega MN, Shahbandar S, Tarbox JA. Approaches to studying the pathogenesis of asthma. The Southwest Respiratory and Critical Care Chronicles 2022;10(42):7-11

From: Department of Internal Medicine, Texas Tech University Health Sciences Center, Lubbock, Texas

Submitted: $9 / 3 / 2021$

Accepted: 1/8/2022

Conflicts of interest: none

This work is licensed under a Creative Commons Attribution-ShareAlike 4.0 International License.

\section{REFERENCES}

1. Popović-Grle S, Štajduhar A, Lampalo M, et al. Biomarkers in different asthma phenotypes. Genes (Basel) 2021;12(6): 801. doi:10.3390/genes12060801.

2. Perlikos F, Hillas G, Loukides S. Phenotyping and endotyping asthma based on biomarkers. Curr Top Med Chem 2016;16(14):1582-1586.

3. Agache I, Akdis CA. Precision medicine and phenotypes, endotypes, genotypes, regiotypes, and theratypes of allergic diseases. J Clin Inves 2019;129(4):1493-150.

4. Samitas K, Zervas E, Gaga M. T2-low asthma, Current Opinion in Pulmonary Medicine 2017:23(1):48-55.

5. Pirmohamed M. Pharmacogenetics and pharmacogenomics. Br J Clin Pharmacol 2001;52:345-7. 
6. What is pharmacogenomics? https://ghr.nlm.nih.gov/primer/ genomicresearch/pharmacogenomics. Accessed 4-5-2019.

7. Tse SM, Tantisira K, Weiss ST. The pharmacogenetics and pharmacogenomics of asthma therapy. Pharmacogenomics J 2011;11:383-92.

8. Drazen JM, Silverman EK, Lee TH. Heterogeneity of therapeutic responses in asthma. Br Med Bull 2000;56:1054-70.

9. Hernandez-Pacheco N, Farzan N, Francis B, et al. Genomewide association study of inhaled corticosteroid response in admixed children with asthma. Clin Exp Allergy 2019;49(6): 789-798.

10. Perez-Garcia J, Espuela-Ortiz A, Lorenzo-Diaz F, et al. Pharmacogenetics of pediatric asthma: current perspectives. Pharmgenomics Pers Med. 2020;13:89-103.

11. Weidner J, Ekerljung L, Malmhäll C, et al. Circulating microRNAs correlate to clinical parameters in individuals with allergic and non-allergic asthma. Respir Res 2020;21(1): 107. doi:10.1186/s12931-020-01351-x.

12. Montuschi P, Barnes PJ. Analysis of exhaled breath condensate for monitoring airway inflammation. Trends Pharmacol Sci 2002;23:232-7.
13. Kazani S, Planaguma A, Ono E, et al. Exhaled breath condensate eicosanoid levels associate with asthma and its severity. J Allergy Clin Immunol 2013;132:547-53.

14. Sinha A, Desiraju K, Aggarwal K, et al. Exhaled breath condensate metabolome clusters for endotype discovery in asthma. J Transl Med 2017;15:262.

15. Brinkman $P$, Wagener $A H$, Hekking $P P$, et al. Identification and prospective stability of electronic nose (eNose)derived inflammatory phenotypes in patients with severe asthma. J Allergy Clin Immunol 2019 May;143(5):18111820 .

16. Kaur R, Chupp G. Phenotypes and endotypes of adult asthma: Moving toward precision medicine. J Allergy Clin Immunol 2019 Jul;144(1):1-12.

17. Richards LB, Neerincx AH, van Bragt JJMH, et al. Biomarkers and asthma management: analysis and potential applications. Curr Opin Allergy Clin Immunol 2018 Apr;18(2): 96-108.

18. Lim HF, Nair P. Airway inflammation and inflammatory biomarkers. Semin Respir Crit Care Med 2018 Feb;39(1): 56-63. 